\title{
Reusable Launch Vehicle Attitude Control Using A Time-Varying Sliding Mode Control Technique
}

\author{
Yuri. B. Shtessel ${ }^{1}$, J. Jim Zhu ${ }^{2}$ and Dan Daniels ${ }^{3}$ \\ 1,3 Department of Electrical and Computer Engineering \\ The University of Alabama in Huntsville, Huntsville, AL 35899 \\ Phone: (256)-824-6164, Fax: (256)-824-8603, \\ E-mail: shtessel@ece.uah.edu \\ ${ }^{2}$ School of Electrical Engineering and Computer Science \\ 353 Stocker Center \\ The Ohio University, Athens, $\mathrm{OH} 45701$ \\ Phone: (740) 597-1506, Fax: (740) 593-0007 \\ E-mail: zhu@homer.ece.ohiou.edu
}

Index Terms: Reusable Launch Vehicle Control, Sliding Mode Control, Time-Varying Control

\begin{abstract}
In this paper we present a time-varying sliding mode control (TVSMC) technique for reusable launch vehicle (RLV) attitude control in ascent and entry flight phases. In ascent flight the guidance commands Euler roll, pitch and yaw angles, and in entry flight it commands the aerodynamic angles of bank, attack and sideslip. The controller employs a body rate inner loop and the attitude outer loop, which are separated in time-scale by the singular perturbation principle. The novelty of the TVSMC is that both the sliding surface and the boundary layer dynamics can be varied in real time using the PD-eigenvalue assignment technique. This salient feature is used to cope with control command saturation and integrator windup in the presence of severe disturbance or control effector failure, which enhances the robustness and fault tolerance of the controller. The TV-SMC ascent and descent designs are currently being tested with high fidelity, 6-DOF dispersion simulations. The test results will be presented in the final version of this paper.
\end{abstract}

1 Corresponding Author, Department of Electrical and Computer Engineering, University of Alabama in Huntsville. 2 School of Electrical Engineering and Computer Science, Ohio University.

3 Department of Electrical and Computer Engineering, University of Alabama in Huntsville. 


\section{Introduction}

Flight control of both current and future reusable launch vehicles (RLV) in ascent and descent modes involves attitude maneuvering through a wide range of flight conditions, wind disturbances, and plant uncertainties including aerodynamic surfaces and engine failures. The baseline RLV flight control system that was designed for the X-33 technology demonstration sub-orbital launch vehicle employs a variable structure PID control law [1] with gain scheduling. This requires four gains per channel that are looked up from a table as a function of relative velocity during ascent flight. Depending on the flight trajectory, each gain table can have as many as 25 values, so potentially 300 gain values must be stored in the on board computer for nominal flight. In case of one engine failure, or Power Pack Out (PPO), alternate sets of gain tables are used, depending on the flight time when the failure occurred. Provisions are made for 25 possible PPO times, or 25 sets of PID tables. This amounts to another 7500 values to be stored, or a total of 7800 values to provide gains for the nominal and engine failed cases during ascent flight. The reason for so many gain tables is because the control system design relies on linear analysis and perturbation theory at specific design points along the trajectory. This method is well established and has been used in many launch vehicle control system designs. Satisfactory control performance is ensured as long as the vehicle performance and operating conditions are relatively close to the design points. A robust flight control algorithm that would accommodate different trajectories and aerodynamic surface and engine failures without gain scheduling would be an improvement over the RLV current flight control technology. Sliding Mode Controller (SMC) is an attractive robust control algorithm for the RLV ascent and descent flight controller designs because of its inherent insensitivity and robustness to plant uncertainties and external disturbances [2-4]. Such a robust controller [5,6] would reduce risk and drastically decrease the amount of time spent in pre-flight analysis, thus reducing cost.

In this work we present a time-varying sliding mode controller (TV-SMC) design technique. The RLV fixed-gain (FG) SMC two-loop structure (Fig. 1), which is presented in the work [5,6], is employed in this work for the TV-SMC design. 


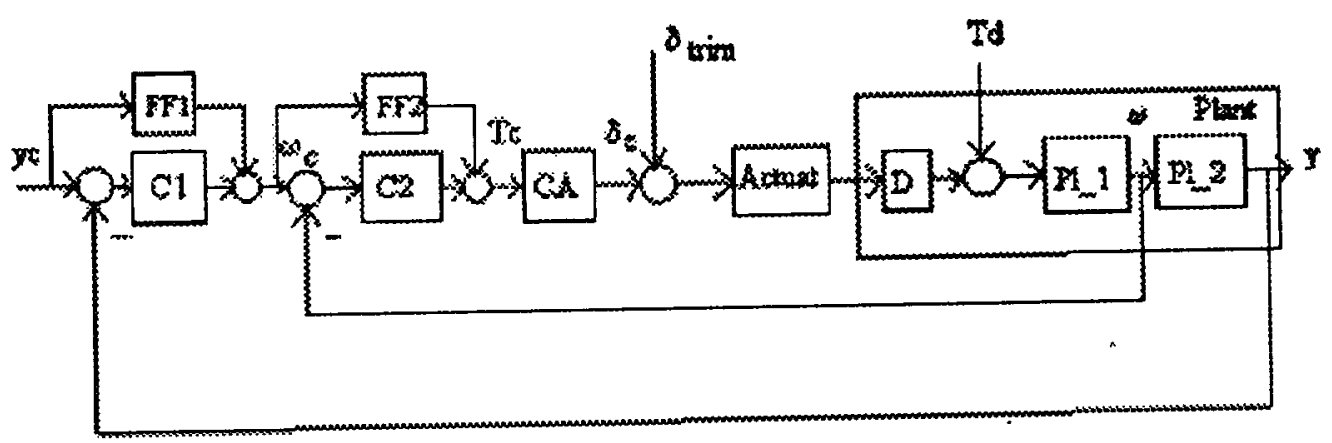

Fig. 1 The RLV SMC two-loop structure

In the outer loop, the kinematics equation of angular motion is used with the outer loop SMC to generate the angular rate profiles as virtual control inputs to the inner loop. In the inner loop, a suitable inner loop SMC is designed so that the commanded angular rate profiles are tracked. The inner loop SMC produces roll, pitch and yaw torque commands, which are allocated into end-effector deflection commands. Multiple time scaling (multiple-scale) is defined as the time-constant separation between the two loops. That is, the inner loop compensated dynamics is designed to be faster than the outer loop dynamics. The resulting multiple-scale two-loop SMC, with optimal torque allocation, causes the angular rate and the Euler angle (ascent) or aerodynamic angle (descent) tracking errors to be constrained to linear de-coupled homogeneous vector valued differential equations with desired eigenvalues.

The TV-SMC design technique is developed for improved robustness in the presence of control effector saturation and tolerance of control effector failure. Any partial or complete failure of control actuators and effectors can be inferred from saturation of one or more commanded control signals generated by the controller. The saturation causes a reduction in the effective gain, or bandwidth of the feedback loop. The controller cannot tell nor does it care whether control command saturation is due to wind gust or a stuck effector, because it only computes the required torque to eliminate the tracking error. A truly adaptive controller should be able to do this even if the vehicle has lost significant control authority due to control effector failure. It is up to the combination of the control allocation and the control effectors to realize the required torque. In order to maintain stability, the bandwidth of the nominal (reduced-order) system will be reduced accordingly using a time-varying bandwidth PD-eigenstructure 
assignment technique $[7,8]$. The presented TV-SMC fault-tolerant technique automatically handles momentary saturations and integrator windup caused by excessive disturbances, guidance command or dispersions under normal vehicle conditions.

The TV-SMC ascent and descent designs are currently being tested with high fidelity, 6DOF dispersion simulations. The test results will be presented in the final version of this paper.

\section{RLV Attitude Control Problem Formulation}

The rigid body equations of motion for an RLV is given by (1)-(5)

$$
\begin{aligned}
\left(\mathbf{J}_{0}+\Delta \mathbf{J}\right) \omega= & -\Omega\left(\mathbf{J}_{0}+\Delta \mathbf{J}\right) \omega+(\mathbf{I}+\mathbf{E}) \mathbf{T}+\mathbf{d}, \\
\dot{\gamma}=\mathbf{R}(\gamma) \omega & \\
\Omega & =\left[\begin{array}{ccc}
0 & -\omega_{3} & \omega_{2} \\
\omega_{3} & 0 & -\omega_{1} \\
-\omega_{2} & \omega_{1} & 0
\end{array}\right]
\end{aligned}
$$

where the rotational matrix $R(\gamma)$ for ascent is given by

$$
\mathbf{R}(\gamma)=\left[\begin{array}{lll}
1 & \tan \theta \sin \varphi & \tan \theta \cos \varphi \\
0 & \cos \varphi & -\sin \varphi \\
0 & \frac{\sin \varphi}{\cos \theta} & \frac{\cos \varphi}{\cos \theta}
\end{array}\right], \gamma=\left[\begin{array}{lll}
\varphi & \theta & \psi
\end{array}\right]^{T}
$$

and the $R(\gamma)$ for entry

$$
\mathbf{R}(\gamma)=\left[\begin{array}{lll}
\cos \alpha & 0 & \sin \alpha \\
0 & 1 & 0 \\
\sin \alpha & 0 & -\cos \alpha
\end{array}\right], \quad \gamma=\left[\begin{array}{lll}
\varphi_{b} & \alpha & \beta
\end{array}\right]^{T}
$$

The control problem for the RLV in ascent and descent modes is to determine the control torque command vector $\mathbf{T}$ such that the commanded orientation angle profiles $\gamma_{c}$ are robustly asymptotically followed in the presence of bounded disturbance torque $d$, the RLV inertia 
variations $\Delta \mathbf{J}$ and aerodynamic surface and engine failures that are described by the uncertain matrix $\Delta \mathbf{D}(),. \mathbf{E}=\Delta \mathbf{D}(.) D^{\prime \prime}, \mathbf{D}^{\prime \prime}()=.\mathbf{D}(.)^{\mathrm{T}}\left[\mathbf{D}(.) \mathbf{D}(.)^{\mathrm{r}}\right]^{-1}, \mathbf{D}($.$) is a nominal sensitivity matrix.$

\section{Summary of the Smooth Multiple-loop Fixed-Gain Sliding Mode Control}

The smooth multiple-loop fixed-gain sliding mode controller is designed as follows $[5,6]$ :

- The outer loop smooth fixed-gain SMC generates body rate commands

$$
\begin{aligned}
& \omega_{c}=\mathbf{R}^{-1}(\gamma)\left[\dot{\gamma}_{c}+\mathbf{K}_{1} \gamma_{e}\right]+\mathbf{R}^{-1}(\gamma) \mathbf{K}_{2} \sigma \\
& \sigma=\gamma_{c}+\mathbf{K}_{1} \int_{0}^{1} \gamma_{e} d \tau, \sigma \in \mathbf{R}^{3}, \gamma_{e}=\gamma_{c}-\gamma
\end{aligned}
$$

or

$$
\omega_{c}=\mathbf{R}^{-1}(\gamma)\left[\dot{\gamma}_{c}+\left(\mathbf{K}_{1}+\mathbf{K}_{2}\right) \gamma_{c}+\mathbf{K}_{2} \mathbf{K}_{1} \int_{0}^{t} \gamma_{e} d \tau\right]
$$

that provide the following outer loop compensated dynamics (given the $\omega_{c}$ profile is tracked perfectly in the inner loop):

$$
\ddot{\gamma}_{e}+\left(\mathbf{K}_{1}+\mathbf{K}_{2}\right) \dot{\gamma}_{e}+\left(\mathbf{K}_{2} \mathbf{K}_{1}\right) \gamma_{e}=0
$$

- The inner loop smooth fixed-gain SMC generates control torque commands

$$
\begin{aligned}
& \mathbf{T}=\mathbf{J}_{0} \dot{\omega}_{c}+\mathbf{J}_{0} \mathbf{K}_{3} \omega_{e}+\Omega \mathbf{J}_{0} \omega+\mathbf{J}_{0} \mathbf{K}_{4} s \\
& s=\omega_{e}+\mathbf{K}_{3} \int_{0}^{t} \omega_{e} d \tau, \quad s \in \mathbf{R}^{3}, \quad \omega_{e}=\omega_{c}-\omega
\end{aligned}
$$

or

$$
\mathbf{T}=\mathbf{J}_{0} \dot{\omega}_{c}+\Omega \mathbf{J}_{0} \omega_{c}+\left(\mathbf{J}_{0} \mathbf{K}_{3}-\Omega \mathbf{J}_{0}+\mathbf{J}_{0} \mathbf{K}_{4}\right) \omega_{\varepsilon}+\mathbf{J}_{0} \mathbf{K}_{4} \mathbf{K}_{3} \int_{0}^{t} \omega_{\varepsilon} d \tau
$$

that provide the following inner loop compensated dynamics (given the $\mathbf{T}$ profile is allocated perfectly into commands to actuator deflections):

$$
\ddot{\omega}_{e}+\left(\mathbf{K}_{3}+\mathbf{K}_{4}\right) \dot{\gamma}_{e}+\left(\mathbf{K}_{4} \mathbf{K}_{3}\right) \gamma_{e}=0
$$


- Writing inner and outer loop tracking error equations in a damping factor/natural frequency format

$$
\ddot{x}+2 \xi \omega_{n} \dot{x}+\omega_{n}^{2} x=0
$$

it is easy to calculate elements of diagonal matrices $K_{1}, K_{2}, K_{3}$ and $K_{4}$ to provide given damping factors (usually all are equal to 1.1 or so) and natural frequencies that provide for a sufficient time-scale separation between the control loops to eqs. (9) and (13) for inner and outer loop compensated tracking dynamics.

- Commands to the actuator deflections can be calculated as follows

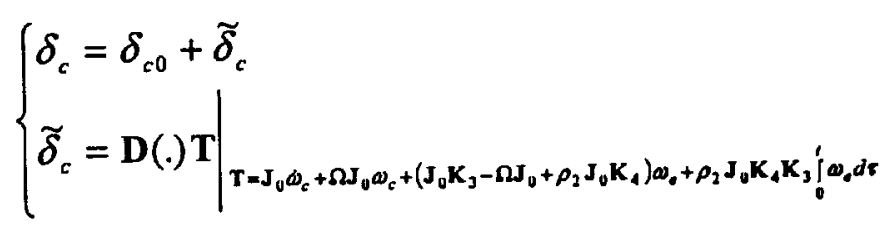

where $\delta_{c 0}$ is a bias command that should trim the RLV, D(.) is a sensitivity matrix (for a feedforward control allocation case).

\section{Smooth Multiple-loop Time-Varying Sliding Mode Control}

Motivations. The elements of the command torque vector (12) are limited by physical abilities of the RLV flight control system that implies the following limits to elements of the vector $\mathbf{T}$ :

$$
\begin{gathered}
a_{i}(.) \leq T_{i} \leq b_{i}(.), \quad i=\overline{1,3} . \\
a_{i}^{\prime}(.) \leq \frac{d}{d t} T_{i} \leq b_{i}^{\prime}(.), \quad i=\overline{1,3}
\end{gathered}
$$

where $a_{i}(),. b_{i}(),. a_{i}^{\prime}(),. b_{i}^{\prime}($.$) depend on the RLV current flying conditions including Mach$ number, dynamic pressure and trim conditions. Designing the time-varying SMC inequalities (17) could be originally out of consideration.

The components of the vector $T$ that depend on body rate tracking profile, $\omega_{c}$, and its derivative, $\omega_{c}$, usually have the largest amplitudes among the others. So, the following idea is proposed: if the inequalities (16) and/or (17) are about to be violated we have to start reducing 
magnitudes of the terms $\omega_{c}$ and $\omega_{c}$ to prevent actuators from saturation. It will be achieved via real time adjusting bandwidths of inner and outer flight control loops. This procedure requires application of time-varying linear control technique $[7,8]$ that is incorporated into the timevarying sliding mode controller design.

The following outer, $\omega_{c}$, and inner, $\mathbf{T}$, continuous, time-varying sliding mode control (TV-SMC) laws are designed

$$
\begin{aligned}
& \omega_{c}=\mathbf{R}^{-1}(\gamma)\left[\dot{\gamma}_{c}+\mathbf{K}_{1}(t) \gamma_{e}\right]+\mathbf{R}^{-1}(\gamma) \mathbf{K}_{2}(t) \sigma \\
& \mathbf{T}=\mathbf{J}_{0} \dot{\omega}_{c}+\mathbf{J}_{0} \mathbf{K}_{3}(t) \omega_{e}+\Omega \mathbf{J}_{0} \omega+\mathbf{J}_{0} \mathbf{K}_{4}(t) s
\end{aligned}
$$

where the outer, $\sigma$, and inner, $s$, sliding quantities are identified

$$
\begin{aligned}
& \sigma=\gamma_{e}+\left(\mathbf{K}_{1}(t)-\mathbf{K}_{2}(t)^{-1} \dot{\mathbf{K}}_{1}(t)\right) \int_{0}^{t} \gamma_{e} d \tau, \quad \sigma \in \mathbf{R}^{3}, \quad \gamma_{e}=\gamma_{c}-\gamma \\
& s=\omega_{e}+\left(\mathbf{K}_{3}(t)-\mathbf{K}_{4}(t)^{-1} \dot{\mathbf{K}}_{3}(t)\right) \int_{0}^{t} \omega_{e} d \tau, \quad s \in \mathbf{R}^{3}, \quad \omega_{e}=\omega_{c}-\omega
\end{aligned}
$$

Remark. One can observe an apparent difference between the eqs. (20), (21) and eqs. (7), (11). These equations become identical if $\mathbf{K}_{1}=$ const and $\mathbf{K}_{3}=$ const .

The diagonal elements of the matrices $K_{2}(t)$ and $K_{4}(t)$ determines the time-varying thickness of the boundary layers of the outer and inner loops, respectively. Once $K_{2}(t)$ and $K_{4}(t)$ are fixed, $K_{1}(t)$ and $K_{3}(t)$ define the dynamics of the sliding modes of the outer and inner loops, respectively. When these controller gains are chosen in a certain manner as defined below, they can be used to define the desired time-varying bandwidths of the outer and inner loops. These bandwidths can be adjusted in real time to prevent actuators from saturation. The corresponding bandwidths or natural frequency of the compensated loop dynamics are to be decreased if the actuator command is close to saturation and are to be increased otherwise. 


\section{Controller Design and Stability Assessment: Nominal Case}

In the nominal case where $E=0, \Delta J=0, d=0$, the mathematical model of the RLV in eqs. (1)-(5) is completely known. For the outer loop, substituting eqs. (18) and (20) into eq. (2) and differentiating eq. (2) we obtained the compensated outer loop error dynamics

$$
\ddot{\tilde{\gamma}}_{e}+\left(\mathbf{K}_{1}(t)+\mathbf{K}_{2}(t)\right) \dot{\tilde{\gamma}}_{e}+\left(\mathbf{K}_{1}(t) \mathbf{K}_{2}(t)-\dot{\mathbf{K}}_{1}(t)\right) \tilde{\gamma}_{e}=\mathbf{R} \dot{\tilde{\omega}}_{e}, \quad \tilde{\gamma}_{e}=\int_{0}^{t} \gamma_{e}(\tau) d \tau
$$

Assume that the transient response in the inner, "faster", loop is stable and $\omega=\omega_{c}$ in eqs. (2) and (18). Then the tracking error $\widetilde{\omega}_{c}$ is stabilized in the inner loop at zero. Assuming $\dot{\varpi}_{c}=0$ the equation (22) can be rewritten in a homogeneous format

$$
\ddot{\gamma}_{e}+\left(\mathbf{K}_{1}(t)+\mathbf{K}_{2}(t)\right) \dot{\tilde{\gamma}}_{e}+\left(\mathbf{K}_{1}(t) \mathbf{K}_{2}(t)-\dot{\mathbf{K}}_{1}(t)\right) \ddot{\gamma}_{e}=0
$$

Since the matrices $K_{1}(t)$ and $K_{2}(t)$ are diagonal, eq. (23) can be rewritten in a scalar format

$$
\ddot{\tilde{\gamma}}_{e i}+\left(k_{1 i}(t)+k_{2 i}(t)\right) \dot{\tilde{\gamma}}_{e i}+\left(k_{1 i}(t) k_{2 i}(t)-\dot{k}_{1 i}(t)\right) \widetilde{\gamma}_{e t}=0, \forall i=\overline{1,3}
$$

The series D-eigenvalues of LTV differential equations (24) can be identified as follows

$$
\lambda_{1 i}(t)=-k_{1 i}(t), \quad \lambda_{2 i}(t)=-k_{2 i}(t), \forall i=\overline{1,3} .
$$

In order to assign values for the bandwidth (cut-off frequency or natural frequency) and damping factor for the compensated dynamics of the outer loop eqs. (24) are rewritten in a format

$$
\ddot{\tilde{\gamma}}_{e i}+\left(2 \xi_{i} \omega_{\mathrm{ni}}(t)-\frac{\dot{\omega}_{\mathrm{ni}}(t)}{\omega_{\mathrm{ni}}(t)}\right) \dot{\tilde{\gamma}}_{e i}+\omega_{\mathrm{ni}}^{2}(t) \tilde{\gamma}_{c i}=0, \xi_{i}>1 \quad \forall i=\overline{1,3}
$$

Then the so-called "PD-eigenvalue" can be identified as follows:

$$
\begin{aligned}
& \Lambda_{1}(t)=\left(-\xi_{i}+\sqrt{\xi_{i}^{2}-1}\right) \omega_{\mathrm{ni}}(t) \\
& \Lambda_{2}(t)=\left(-\xi_{i}-\sqrt{\xi_{i}^{2}-1}\right) \omega_{\mathrm{ni}}(t)
\end{aligned}
$$


Note that for time-varying $\omega_{n i}(t)$, the relations between the sign of the radicals and the index of $\Lambda$ must be maintained. Then the SD-eigenvalues are related to the PD-eigenvalues as follows

$$
\lambda_{1 i}(t)=\Lambda_{1}(t)=\left(-\xi_{i}+\sqrt{\xi_{i}^{2}-1}\right) \omega_{n i}(t)=-k_{1 i}(t), \forall i=\overline{1,3}
$$

or

$$
k_{1 i}(t)=\left(\xi_{i}-\sqrt{\xi_{i}^{2}-1}\right) \omega_{\mathrm{ni}}(t)
$$

and

$$
\lambda_{2 i}(t)=\Lambda_{2 i}(t)+\frac{\dot{\omega}_{\mathrm{ni}}(t)}{\omega_{\mathrm{ni}}(t)}=\left(-\xi_{i}-\sqrt{\xi_{i}{ }^{2}-1}\right) \omega_{\mathrm{ni}}(t)+\frac{\dot{\omega}_{\mathrm{ni}}(t)}{\omega_{\mathrm{ni}}(t)}=-k_{2 i}(t)
$$

or

$$
k_{2 i}(t)=\left(\xi_{i}+\sqrt{\xi_{i}^{2}-1}\right) \omega_{\mathrm{ni}}(t)-\frac{\omega_{\mathrm{ni}}(t)}{\omega_{\mathrm{ni}}(t)}
$$

Thus, given $\xi_{i}$ and $\omega_{\mathrm{ni}}(t)$ the corresponding time-varying coefficients in the sliding surface (8) can be computed using eqs. (30) and (32).

\section{Remarks.}

1. The outer loop dynamics are obviously decoupled and the stability is guranteed by the PDeigenvalues for $\xi_{i}>1$ and $\omega_{n i}(t)>0, \forall t \geq t_{0}$, which can be achieved by the choice of diagonal time-varying matrices $K_{1}(t), K_{2}(t)$ according to (30) and (32).

2. The gain matrices $K_{1}(t), K_{2}(t)$ must be selected in accordance with (30) and (32) with $\xi_{i}>1$ in order to to make $\sigma$-dynamics faster then $\tilde{\gamma}_{e}$-dynamics. The time scale separation increases with $\xi_{i}$. Note that (30) and (32) are for constant $\xi_{i}$ only, and as such $\xi_{i}$ should not be adjusted in real time. The PD-eigenvalue synthesis formulas for time-varying $\xi_{i}(t)$ is available, but it is much more complex. 
The inner loop compensated error dynamics are described by the eqs. (1), (3), (19) and (21), which can be combined as

$$
\ddot{\tilde{\omega}}_{e}+\left(K_{3}(t)+K_{4}(t)\right) \dot{\tilde{\omega}}_{e}+\left(K_{3}(t) K_{4}(t)-\dot{K}_{3}(t)\right) \widetilde{\omega}_{e}=0, \quad \tilde{\omega}_{e}=\int_{0}^{1} \omega_{e}(\tau) d \tau
$$

Eq. (33) can be rewritten in a scalar "damping factor-natural frequency" format

$$
\ddot{\tilde{\omega}}_{e i}+\left(2 \hat{\xi}_{i} \hat{\omega}_{\mathrm{ni}}(t)-\frac{\dot{\hat{\omega}}_{\mathrm{ni}}(t)}{\hat{\omega}_{\mathrm{ni}}(t)}\right) \dot{\tilde{\omega}}_{e l}+\hat{\omega}_{\mathrm{ni}}^{2}(t) \tilde{\omega}_{e i}=0, \hat{\xi}_{i}>1 \forall i=\overline{1,3}
$$

Eqs. (33) and (34) is a full anology to eqs. (24) and (26). So, the following coefficients can be identified by analogy

$$
\begin{gathered}
k_{3 i}(t)=\left(\xi_{i}-\sqrt{\xi_{i}^{2}-1}\right) \hat{\omega}_{\mathrm{ni}}(t) \\
k_{4 i}(t)=\left(\xi_{i}+\sqrt{\xi_{i}^{2}-1}\right) \hat{\omega}_{\mathrm{ni}}(t)-\frac{\dot{\omega}_{\mathrm{ni}}(t)}{\omega_{\mathrm{ni}}(t)}
\end{gathered}
$$

Now, given $\hat{\xi}_{i}$ and $\hat{\omega}_{\mathrm{ni}}(t)$ the corresponding time-varying coefficients in the sliding surface (21) can be computed concerning eqs. (18) and (19) $\forall i=\overline{1,3}$.

Remark. The same comments for $K_{1}(t), K_{2}(t)$ following (30) and (32) are applicable to $K_{3}(t), K_{4}(t)$ in relation to (35) and (36), and the $s$-dynamics and $\omega_{e}$-dynamics.

\section{Stability Assessment: Perturbed Case}

In the perturbed case assume $E \neq 0, \Delta J \neq 0, d \neq 0$, unknown but bounded, i.e. the math model of the RLV in eqs. (1)-(5) is not completely known. For the outer loop, again assume that the transient in the inner "faster" loop is stable and $\omega=\omega_{c}$ in eqs. (2) and (18). Then the perturbed case coinsides with the nominal case, since eqs. (2), (4) and (5) do not contain uncertaintes and disturbances. 
The inner loop error compensated dynamics are described by the eqs. (1), (3), (19) and (21). In the perturbed case this is

$$
\ddot{\tilde{\omega}}_{e}+\left(K_{3}(t)+K_{4}(t)\right) \dot{\tilde{\omega}}_{e}+\left(K_{3}(t) K_{4}(t)-\dot{K}_{3}(t)\right) \widetilde{\omega}_{e}=F\left(\widetilde{\omega}_{e}, \dot{\varpi}_{e}, \ddot{\tilde{\omega}}_{e}, t\right)
$$

where $F\left(\omega_{e}, \omega_{e}, \omega_{e}, t\right)$ depends on perturbation terms. The closed-loop stability of the perturbed system can be justified by the well-known results from the Lyapunov analysis [9].

Theorem. Given a perturbed linear time-varying dynamic system

$$
\dot{x}=A(t) x+F(x, t)
$$

where $F(x, t) \leq \delta$ is a bounded, nonvanishing perturbation, i.e. $F(0, t) \neq 0$, and the matrix $A(t)$ is Hurwitz, i.e. there exists a pair of positive definite matrices $(P(t), Q(t))$ satisfying the Lyapunov equation

$$
\dot{P}(t)+P(t) A(t)+A^{T}(t) P(t)=-Q(t)
$$

such that

$$
\begin{gathered}
c_{1}\|x(t)\|^{2} \leq x^{\mathrm{T}}(t) P(t) x(t) \leq c_{2}\|x(t)\|^{2} \\
x^{\mathrm{T}}(t) Q(t) x(t) \leq-c_{3}\|x(t)\|^{2} \\
\left\|x^{\mathrm{T}}(t) P(t)\right\| \leq c_{4}\|x(t)\|
\end{gathered}
$$

for all $t>t_{0}$, where \|\|$=\sqrt{v^{\mathrm{T}} v}$. For any $0<\theta<1$, define

$$
\delta_{0}(\theta)=\frac{2 c_{3}}{c_{4}} \sqrt{\frac{c_{1}}{c_{2}}} \theta
$$

If $F(x, t) \leq \delta<\delta_{0}$, then every trajectory of the perturbed system is exponentially, ultimately bounded in the sense that for any $r>0$, and $\left\|x\left(t_{0}\right)\right\| \leq r / k$, there exists a $t_{1}>t_{0}$ such that

$$
\left\|x\left(t, t_{0}\right)\right\| \leq k\left\|x\left(t_{0}\right)\right\| \mathrm{e}^{-\lambda\left(t-t_{0}\right)}, \quad t_{0} \leq t<t_{1},
$$




$$
\left\|x\left(t, t_{0}\right)\right\| \leq b, \quad t \geq t_{1}
$$

where

$$
k=\sqrt{\frac{c_{2}}{c_{1}}}, \quad \lambda=\frac{(1-\theta) c_{3}}{2 c_{2}}, \quad b=\frac{\delta}{\delta_{0}(\theta)} r
$$

\section{Summary and Conclusions}

The time-varying sliding mode controller (TV-SMC) design algorithm is developed for the $2^{\text {nd }}$ generation reusable launch vehicle in ascend and descend modes. In order to maintain stability, the bandwidth of the nominal (reduced-order) system is reduced accordingly using a time-varying bandwidth PD-eigenstructure assignment technique. The presented TV-SMC faulttolerant technique automatically handles momentary saturations and integrator windup caused by excessive disturbances, guidance command or dispersions under normal vehicle conditions. The TV-SMC algorithm has been coded for the X-33 technology demonstration vehicle in ascent and descent modes. The results of simulations of the designed TV-SMC will be presented and discussed in the final version of the paper.

\section{References}

1. Hall, C. E., Hodel, A. S., and Hung, J. Y., "Variable Structure PID Control to Prevent Integral Windup", Proceedings of the $31^{\text {st }}$ Southeastern Symposium on System Theory, IEEE, 1999, pp. 169-173.

2. DeCarlo, R. A., Zak, S. H., and Matthews, G. P. "Variable structure control of nonlinear multivariable systems: a tutorial," IEEE Proceedings, Vol. 76, 1988, pp. 212-232.

3. Utkin, V. I., Sliding Modes in Control and Optimization, Berlin, Springer - Verlag, 1992, pp. 15-82. 
4. Hung, J. Y., Gao, W., and Hung, J. C., "Variable Structure Control: A Survey," IEEE Transactions on Industrial Electronics, Vol. 40, No.1, 1993, pp. 2-21.

5. Y. Shtessel, C. Hall, and M. Jackson, "Reusable Launch Vehicle Control in Multiple Time Scale Sliding Modes," AIAA Journal on Guidance, Control, and Dynamics, Vol. 23, No. 6, pp. 1013-1020, 2000.

6. Yuri B. Shtessel and Charles E. Hall, "Multiple time scale sliding mode control of reusable launch vehicles in ascent and descent modes," Proceedings of American Control Conference, Washington, DC, June 2001.

7. J. Zhu, B. D. Banker and Charles E. Hall, X-33 ascent flight controller design by trajectory linearization - a singular perturbational approach, AIAA-2000-4159, AIAA Guidance, Navigation and Control Conference, Denver, Colorado, Aug. 2000.

8. J. Zhu, A. Scott Hodel, Kerry Funston and Charles E. Hall, X-33 entry flight controller design by trajectory linearization - a singular perturbational approach, Proceedings, American Astronautical Society Guidance and Control Conference, pp. 151-170, Breckenridge, Colorado, Jan. 2001.

9. H. Khalil, Nonlinear Systems, Second Edition, Prentice Hall, 1996. 